Pacific Journal of Mathematics

AICAL PONTS ON RMM-COMPCCT SPACS 


\section{CRITICAL POINTS ON RIM-COMPACT SPACES}

\section{DAVID H. CARLSON}

In this note we prove that all the points of a rim-compact space $X$ at which $X$ is not locally compact are critical points for any local dynamical system defined on $X$. When a local system is global this result is obtained by extending the global system $\pi$ on $X$ to a global system $\rho$ on the Freudenthal compactification $Y$ of $X$, then showing that $Y-X$ is a critical set for $\rho$, and, finally, observing that $\overline{Y-X}$ contains all the points of $X$ at which $X$ is not locally compact. This weaker result will appear in the author's doctoral dissertation and requires the use of general extension theorems proven there. For this paper, we isolate those parts of the thesis which are pertinent to our theorem.

Definition 1. [1]. A (continuous) local dynamical system on a topological space $Z$ is an object $\pi$ satisfying the following conditions: ( $R$ is the set of real numbers with the usual topology)

(1) $\pi$ is a continuous partial map from $Z \times R$ into $Z$.

(2) For every $z$ in $Z$ there are (bounds) $\alpha_{z}$ and $\omega_{z}$ such that $-\infty \leqq \alpha_{z}<0,0<\omega_{z} \leqq+\infty$ and $\pi(z, t)$ is defined if and only if $t$ is in $\left(\alpha_{z}, \omega_{z}\right)$.

(3) The domain of $\pi$ is open in $Z \times R$.

(4) For each $z$ in $Z, \pi(z, 0)=z$.

(5) When $\pi(z, t)$ is defined, $\pi\left(\pi(z, t), t^{\prime}\right)=\pi\left(z, t+t^{\prime}\right)$ whenever either side is defined.

A local dynamical system $\pi$ on $Z$ is global if $\alpha_{z}=-\infty$ and $\omega_{z}=+\infty$ for all $z \in Z$. Condition (4) is called the initial value condition, and condition (5) the additive condition. Because of the additive condition, it has become conventional to write $\pi(z, t)$ as $z \pi t$. Thus the equality in condition (5) is written $(z \pi t) \pi t^{\prime}=z \pi\left(t+t^{\prime}\right)$. To prove that a point $z$ is a critical point of $\pi$, it suffices to show there is an $\varepsilon>0$ such that $z=z \pi t$ for all $t \in[0, \varepsilon)$.

Definition 2. A topological space $X$ is said to be rim-compact if and only if it is $T_{2}$ and each point of $X$ has a fundamental system of neighborhoods with compact boundaries.

In this paper $X$ denotes a rim-compact space and $Y$ denotes the Freudenthal compactification of $X$, ([2], p. 111). $Y$ is $T_{2}$ and every point in $Y$ has a fundamental system of neighborhoods with compact boundaries entirely in $X$. These and compactness are the only properties of $Y$ that will be used. Convergence of a net $\left\{y_{i}\right\}$ indexed 
by the directed set $I$ is written as $\left\{y_{i}\right\} \stackrel{i}{\longrightarrow} y$; thus a net $\left\{y_{j}^{i} \mid j \in J^{i}\right\}$, indexed by $J^{i}$, converging to the point $y_{i}$ is written as $\left\{y_{j}^{i}\right\} \stackrel{j}{\longrightarrow} y_{i}$; and convergence of the composite net is written as $\left\{y_{j}^{i}\right\} \stackrel{i j}{\longrightarrow} y$.

THEOREM. For a local dynamical system $\pi$ on a rim-compact space $X$, each point in $X$ which does not have a compact neighborhood is a critical point of $\pi$.

Proof. Consider $X$ as a subspace of its Freudenthal compactification, $Y$. All of our work is done in the space $Y$, with the following notational convention: the letter $x$ denotes an element of $X$, the letter $b$ denotes an element of $Y-X$, and the letter $y$ denotes an element of $Y$ (all of these may be equipped with various indices).

Let $x$ be any point which does not have a compact neighborhood in $X$. There is a net $\left\{b_{i}\right\} \stackrel{i}{\longrightarrow} x$, with an index set $I$, since for any compact neighborhood $N_{x}$ of $x$ we have that $N_{x} \cap X$ is not compact, which implies $N_{x} \cap Y-X \neq \varnothing$. Also, since $X$ is dense in $Y$, for each $b_{i}$ there is a net $\left\{x_{j}^{i}\right\} \stackrel{j}{\longrightarrow} b_{i}$ with index set $J^{i}$. Choose $t>0$ so small that $x \pi t$ is defined, and assume $x \neq x \pi t$. Because $\left\{x_{j}^{i}\right\} \stackrel{i j}{\longrightarrow} x$, eventually $\left\{x_{j}^{i} \pi t\right\}_{i, j}$ is defined. More precisely, there is an antiresidual subset $I^{\prime}$ of $I$ and an antiresidual subset of $J^{i}$ for each $i$ in $I^{\prime}$ such that $\left\{x_{j}^{i} \pi t\right\}$ is defined. Without loss of generality, let $I$ and $J^{i}$ be the antiresidual subsets described above. Again, there must be an antiresidual subset of $I$ for which the net $\left\{x_{j}^{i} \pi t\right\}_{J i}$ does not converge to $b_{i}$. If not, there would be a cofinal subset of $I$ such that $\left\{x_{j}^{i} \pi t\right\} \stackrel{j}{\longrightarrow} b_{i}$ and thus a subnet of $\left\{x_{j}^{i} \pi t\right\} \stackrel{i j}{\longrightarrow} x$, which would imply $x=x \pi t$. Once more, let $I$ be the new antiresidual subset.

Each $b_{i}$ has a neighborhood $N_{i}$ such that some subnet of $\left\{x_{j}^{i} \pi t\right\}_{J^{i}}$ is not in $N_{i}$ and that its boundary $\partial N_{i}$ is compact and contained in $X$. Denote this subnet by $\left\{x_{j}^{i} \pi t\right\}$. From the connectedness of $x_{j}^{i} \pi[0, t]$ there is a $\tau_{j}^{i}$ in $(0, t)$ such that $x_{j}^{i} \pi \tau_{j}^{i} \in \partial N_{i}$. As $\partial N_{i}$ is compact and $\left\{\tau_{j}^{i}\right\}_{J i}$ is bounded, there are subnets $\left\{x_{j}^{i} \pi \tau_{j}^{i}\right\} \stackrel{j}{\longrightarrow} x^{i}$ in $\partial N_{i}$ and $\left\{\tau_{j}^{i}\right\} \stackrel{j}{\longrightarrow} \tau^{i}$. Let $\left\{x_{j}^{i} \pi \tau_{j}^{i}\right\}_{J^{i}}$ and $\left\{\tau_{j}^{i}\right\}_{J^{i}}$ denote these subnets. Since $Y$ is compact and $\left\{\tau^{i}\right\}$ is bounded there are subnets of $\left\{x^{i}\right\} \stackrel{i}{\longrightarrow} y \in Y$ and of $\left\{\tau^{i}\right\} \stackrel{i}{\longrightarrow} \tau \in$ $[0, t]$, again denoted by $\left\{x^{i}\right\}$ and $\left\{\tau^{i}\right\}$. Thus, we have $\left\{x_{j}^{i} \pi \tau_{j}^{i}\right\} \stackrel{i j}{\longrightarrow} y$ and $\left\{\tau_{j}^{i}\right\} \stackrel{i j}{\longrightarrow} \tau$. However, since $x \pi \tau$ is defined and $\left\{x_{j}^{i}\right\} \stackrel{i j}{\longrightarrow} x$, we obtain $y=x \pi \tau$. From condition (3) of Definition 1, this implies there is an $i$ such that $x^{i} \pi\left(-\tau^{i}\right)$ is defined. For this $i,\left\{x_{j}^{i} \pi \tau_{j}^{i}\right\} \stackrel{j}{\longrightarrow} x^{i},\left\{-\tau_{j}^{i}\right\} \stackrel{j}{\longrightarrow}-\tau^{i}$ implies $\left\{\left(x_{j}^{i} \pi \tau_{j}^{i}\right) \pi-\tau_{j}^{i}\right\} \stackrel{j}{\longrightarrow} x^{i} \pi-\tau^{i}$ which is in $X$. But, $\left\{\left(x_{j}^{i} \pi \tau_{j}^{i}\right) \pi-\tau_{j}^{i}\right\}=$ $\left\{x_{j}^{i}\right\} \stackrel{j}{\longrightarrow} b_{i} \in Y-X$. This contradiction yields our assertion. 
COROLlaRY. Let a space $Z$ have a rim-compact but not locally compact open subset. Then every local dynamical system on $Z$ has a critical point.

\section{REFERENCES}

1. O. Hajek, Dynamical systems in the plane, Academic Press, New York, London 1968.

2. J.R. Isbell, Uniform spaces, American Mathematical Society, Providence, Rhode Island, 1964.

3. J. L. Kelley, General topology, D. Van Nostrand, Princeton, New Jersey, 1955.

Received July 1, 1968. This paper was partially supported by NSF Grant GP 8961.

CASE Western Reserve University 




\section{Pacific Journal of Mathematics \\ Vol. 29, No. 1 \\ May, 1969}

Jorge Alvarez de Araya, A Radon-Nikodým theorem for vector and operator

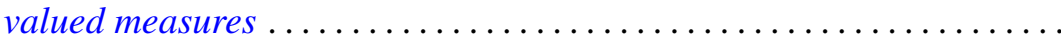

Deane Eugene Arganbright, The power-commutator structure of finite

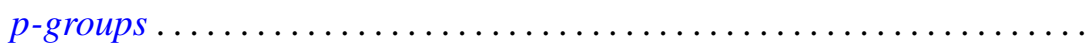

Richard Eugene Barlow, Albert W. Marshall and Frank Proschan, Some

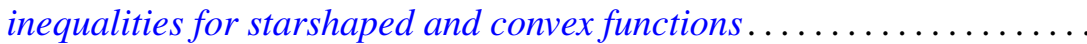

David Clarence Barnes, Some isoperimetric inequalities for the eigenvalues

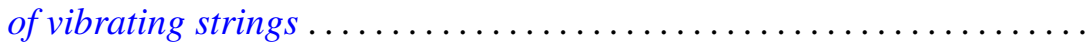

David Hilding Carlson, Critical points on rim-compact spaces ...........

Allan Matlock Weber Carstens, The lattice of pretopologies on an arbitrary

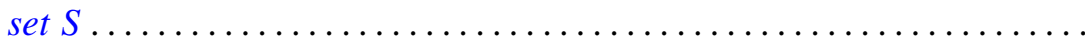

S. K. Chatterjea, A bilateral generating function for the ultraspherical

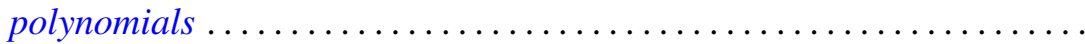

Ronald J. Ensey, Primary Abelian groups modulo finite groups ......... 77

Harley M. Flanders, Relations on minimal hypersurfaces ............ 83

Allen Roy Freedman, On asymptotic density in n-dimensions........... 95

Kent Ralph Fuller, On indecomposable injectives over artinian rings...... 115

George Isaac Glauberman, Normalizers of p-subgroups in finite groups . . . 137

William James Heinzer, On Krull overrings of an affine ring ........... 145

John McCormick Irwin and Takashi Ito, A quasi-decomposable abelian group without proper isomorphic quotient groups and proper isomorphic subgroups.

Allan Morton Krall, Boundary value problems with interior point boundary conditions

John S. Lowndes, Triple series equations involving Laguerre

polynomials

Philip Olin, Indefinability in the arithmetic isolic integers

Ki-Choul Oum, Bounds for the number of deficient values of entire functions whose zeros have angular densities..

R. D. Schafer, Standard algebras ....................

Wolfgang M. Schmidt, Irregularities of distribution. III.

Richard Alfred Tapia, An application of a Newton-like method to the Euler-Lagrange equation 